\section{Revolution vs status quo? Non-intervention strategy of asymptomatic third molars causes harm}

\author{
V. Toedtling ${ }^{* 1}$ and J. M. Yates ${ }^{2}$
}

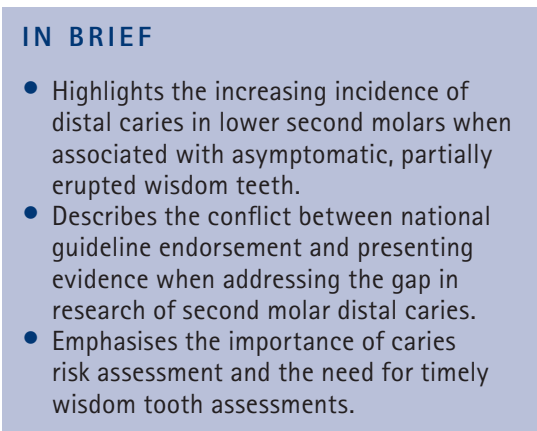

The Faculty of Dental Surgery of the Royal College of Surgeons of England has prompted the National Institute for Health and Care Excellence to urgently re-assess the TA1 guidance on extractions of wisdom teeth and highlighted required amendments to the present version in light of published evidence concerning the harm caused by wisdom tooth retention. This article seeks to address the recent concerns relating the increasing frequency of distal-cervical caries in lower second molar teeth when associated with asymptomatic partially erupted mesial or horizontal impacted mandibular third molars. Such acute angle impactions are classified as partially erupted when one of the third molar cusps breached the epithelial attachment of the distal aspect of the second molar, thus prevents the formation of a gingival seal. At its earliest stage the wisdom tooth appears clinically absent or unerupted, yet histologically the architecture of the gingival epithelium has been disrupted allowing ingress of microbes, demineralisation and succeeding cavitation to take place on the distal aspect of the second molar. We hope to highlight the difficulties faced in addressing this growing clinical problem and encourage clinicians to re-evaluate their own caries risk assessment and caries prevention strategy in relation to mesial and horizontal third molar extractions.

\section{INTRODUCTION}

The Faculty of Dental Surgery of the Royal College of Surgeons of England (FDS RCSEng) has recently published their response to the National Institute for Health and Care Excellence's (NICE) review proposal of the guidelines on wisdom tooth removal. The reply indicates that NICE is reluctant to re-appraise the 2000 guidance and states that existing evidence is not strong enough to necessitate a review of their first ever published guidance document since being founded. In contrast, it is with great interest that the current best available and informative evidence on third molar research has led to significant amendments of international guidance documents in favour of removal in Scandinavia, Germany and the US. The reluctance by NICE to undertake a new consultation is somewhat surprising as there are numerous consultations currently underway in other clinical fields, aiming to provide the

${ }^{1}$ Clinical Lecturer and Honourary Specialist Registrar in Oral Surgery, ${ }^{2}$ Professor of Oral and Maxillofacial Surgery, School of Dentistry, JR Moore Builing, University of Manchester, Oxford Road, Manchester, M13 9PL; ${ }^{*}$ Correspondence to: Dr Verena Toedtling

Email: verena.toedtling@manchester.ac.uk

Tel: +44 (0)1612754383

\section{Refereed Paper}

Accepted 10 June 2015

DOI: 10.1038/sj.bdj.2015.525

${ }^{\circ}$ British Dental Journal 2015; 219: 11-12 most up to date guidance, quality standards and advice on clinical interventions.

\section{USE OF MOST \\ APPROPRIATE EVIDENCE}

The existing guidelines are based on evidence from an assessment report published by Song et al. ${ }^{1}$ in 1999 which refers to research evidence from almost three decades ago, during which time a large number of third molars were prophylactically removed. Moreover, the assessment report documented a very low rate of distal-cervical caries in lower second molars and identified the incidence within a range of $1-4.5 \% .^{2}$ It is now evident that the removal of wisdom teeth in this era could not provide the data or analysis required to truly highlight the incidence of harm that clinicians see today - some 15 years after the recommendation for a non-intervention strategy.

\section{Conflict between research evidence and clinical opinion}

In the UK we have little hope of enhancing our evidence in relation to third molar research with large trials as research funders for the NHS (England) and other UK based research councils have funding priorities that lay elsewhere. ${ }^{3}$ Furthermore, the current clinical reporting systems in place are not sensitive enough to show an accurate picture of the problem, are not in a position to provide outcome data to inform decisions, and therefore a change in clinical practice. Thus, very little consideration is given to the problems faced by an increasing number of clinicians in the post prophylactic removal era. One of these problems is distal-cervical caries within second mandibular molars, which can be particularly insidious in its presentation. In our experience, patients often remain asymptomatic until late in the disease process with extraction of the second molar frequently the only option.

\section{WE WANT THE BEST CARE FOR OUR PATIENTS}

So what impact does this have on our patients when clinical observations and published guidance on the management of third molars are not aligned? Patients now retain their wisdom teeth for longer however, symptoms or pathological changes ultimately emerge and removal is frequently indicated. It has become evident that the NICE guidance is only appropriate for immediate and shortterm decision making and does not take into account the long-term consequences of third molar retention. McArdle et al. ${ }^{4}$ reported that patients with distal-cervical caries in second molars tend to be on average five-years older than patients with other pathological manifestations. This illustrates 
that a significant proportion of our patients will suffer increased risks and complications. Initially, risks are associated with the deliberate retention of third molars. Subsequently, there are also risks related to the removal and of increased morbidity caused by more complex intervention requirements in later years. Furthermore, for some of our patients the effect will be bilateral.

\section{A CASE IN POINT}

Recently a 35-year-old male patient referred by his general dental practitioner (Fig. 1) was understandably frustrated when we communicated our findings and treatment option - namely extraction of the lower left and right second as well as third molar teeth. Originally he was referred to the Oral Surgery Department at the Manchester University Dental Hospital by his general dental practitioner for a third molar assessment. Like many other patients on presentation, the patient was asymptomatic and reported to attend six-monthly dental re-call appointments for many years. Intra-oral examinations revealed an unremarkable dentition, excellent oral hygiene with only minimal staining of the occlusal surfaces of the partially erupted mandibular third molars. A dental panoramic tomograph showed evidence of large carious lesions on the distalcervical aspect of both second mandibular molars, undoubtedly caused by the partially erupted mesio-angular impacted wisdom teeth. The difficulty of cleaning this type of impaction almost guarantees development of large carious lesions in the second molar in the long term. Our own research and analysis of carious second molars in association with partially erupted mandibular third molars demonstrated an alarming incidence of 38\% carious involvement in all referrals received, and when mesial and horizontal angulations were pooled other reported incidence rose to an overwhelming $85 \% .^{5}$

So why is it so difficult to detect these lesions earlier? The issue is multifactorial, but in our experience, many adult patients are not formally risk assessed for caries, particularly around the distal surface of the second molars. Riley et al. ${ }^{6}$ reported that dentists who use a formal caries risk assessment appear to use this information in clinical decision-making, and for individualisation of the caries prevention strategy. Frequently it seems that the risk factor associated with longstanding impacted mandibular third molars is overlooked. Given
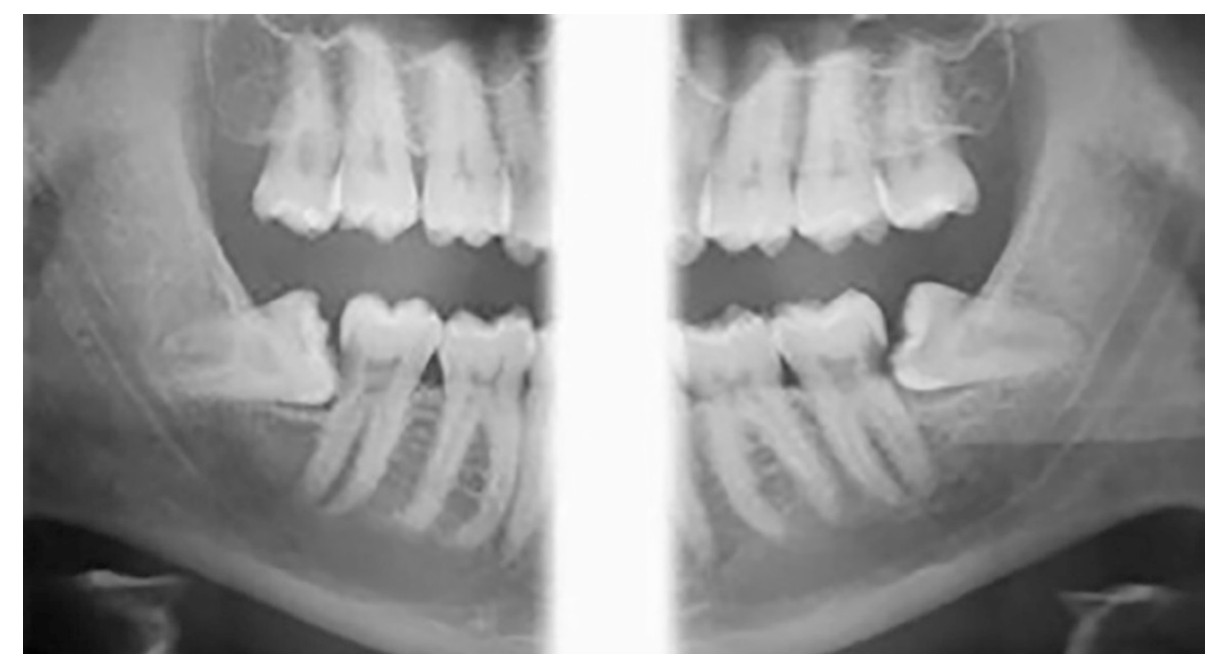

Fig. 1 Dental panoramic tomograph of mandibular mesio-angular impacted wisdom teeth in a 35-year-old with bilateral distal-cervical caries in the adjacent molars

the incidence of this problem patients with mesial and horizontal third molar impactions should be considered at high-risk of developing carious lesions in the distal sites of lower second molars in the medium to long term, and given the difficulty of providing effective preventative treatment for these lesions removal of the adjacent third molars should in our opinion be considered before the initiation of active decay. It should also be acknowledged that patients who do not have active disease or have no previous caries experience are not at low or no risk of developing caries in the second molars, as seen in the presented case. Another issue with early caries detection is that there is no formal caries risk management protocol specifically for partially erupted wisdom teeth, or explicit guidance to assist general dental practitioners on the frequency and effectiveness of screening and preventative measures in order to improve their decision making process in relation to the management of such scenarios. Finally, is it reasonable to expect patients who have asymptomatic partially erupted third molars not to develop decay over a 60-70-year period? A growing body of clinicians feel this is an unrealistic expectation.

\section{A SOLUTION?}

Proponents for the NICE guidance will say just that, they are only guidelines (recommendations for best practice), and that each case should be evaluated on an individual basis. However, given the status afforded to NICE many clinicians, patients, and even those within the legal profession, a more appropriate, readily applicable and accurate reiteration would be NICE standards (specific mandatory controls). So how can patients' quality of care and prognosis of lower second molars in the presence of asymptomatic mesially/horizontally impacted lower third molars be enhanced in this current atmosphere of debate and knowledge that contradicts current guidelines? For now clinicians have no choice but to use the tools and guidance already available as best as reasonably achievable. Namely, a rigorous caries risk assessment, biannual bitewing radiographs, individualised preventative measures and timely assessment of third molars.

Will this have a positive impact on the incidence of distal-cervical caries in lower second molars? We hope for the benefit of our patients.

1. Song F, O'Meara S, Wilson P, Golder S, Kliejnen J. The effectiveness and cost effectiveness of the prophylactic removal of wisdom teeth. Health Technol Assess 2000; 4: 1-55.

2. National Institute for Clinical Excellence. Guidance on the extraction of wisdom teeth. 2000. Online information available at: https://www.nice.org.uk/ guidance/ta1 (accessed June 2015).

3. The Faculty of Dental Surgery of the Royal College of Surgeons of England. FDS response to NICE review 2014 of guidance on the extraction of wisdom teeth, Online information available at https://www.rcseng. ac.uk/fds/policy/documents/nice-review-of-guidanceon-wisdom-teeth-extraction-fds-response-withappendices/view (accessed June 2015).

4. McArdle L W, McDonald F, Jones J. Distal cervical caries in the mandibular second molar: an indication for the prophylactic removal of third molar teeth? Update. Br J Oral Maxillofac Surg 2014; 52: 185-189.

5. Toedtling V. Study of third molar retention - analysis and outcomes of carious second molars. Oral Surg 2014; 7: 116-117.

6. Riley J L 3rd, Gordan V V , Ajmo C T et al. Dentists' use of caries risk assessment and individualised caries prevention for their adult patients: findings from The Dental Practice-Based Research Network. Community Dent Oral Epidemiol 2011; 39: 564-573. 